\title{
Optimal control for fast and high-fidelity quantum gates in coupled superconducting flux qubits
}

\author{
Shang-Yu Huang ${ }^{1,2}$ and Hsi-Sheng Goan ${ }^{1,2, *}$ \\ ${ }^{1}$ Department of Physics and Center for Theoretical Sciences, \\ National Taiwan University, Taipei 10617, Taiwan \\ ${ }^{2}$ Center for Quantum Science and Engineering, \\ and National Center for Theoretical Sciences, \\ National Taiwan University, Taipei 10617, Taiwan
}

(Dated: June 14, 2021)

\begin{abstract}
We apply the quantum optimal control theory based on the Krotov method to implement singlequbit $X$ and $Z$ gates and two-qubit CNOT gates for inductively coupled superconducting flux qubits with fixed qubit transition frequencies and fixed off-diagonal qubit-qubit coupling. Our scheme that shares the same advantage of other directly coupling schemes requires no additional coupler subcircuit and control lines. The control lines needed are only for the manipulation of individual qubits (e.g., a time-dependent magnetic flux or field applied on each qubit). The qubits are operated at the optimal coherence points and the gate operation times (single-qubit gates $<1$ ns; CNOT gates $\sim 2 \mathrm{~ns}$ ) are much shorter than the corresponding qubit decoherence time. A CNOT gate or other general quantum gates can be implemented in a single run of pulse sequence rather than being decomposed into several single-qubit and some entangled two-qubit operations in series by composite pulse sequences. Quantum gates constructed via our scheme are all with very high fidelity (very low error) as our optimal control scheme takes into account the fixed qubit detuning and fixed two-qubit interaction as well as all other time-dependent magnetic-field-induced single-qubit interactions and two-qubit couplings. The effect of leakage to higher energy-level states and the effect of qubit decoherence on the quantum gate operations are also discussed.
\end{abstract}

PACS numbers: 03.67.Lx, 85.25.Cp, 02.30.Yy

* goan@phys.ntu.edu.tw 


\section{INTRODUCTION}

Superconducting Josephson junction devices and circuits are proving to be promising systems for quantum information processing [1, 2]. Due to the great controllability of the qubits and microwaves in the superconducting circuit systems, single-qubit [3 -14$]$, two-qubit [15 23], and three-qubit [24] operations have been experimentally demonstrated. The next natural step is to develop robust, high-fidelity and scalable gates for larger scale quantum computation.

An essential prerequisite for quantum information processing and quantum computation is precise coherent control of quantum systems or quantum bits (qubits). Here, we focus our discussion on high-fidelity quantum control for superconducting flux qubit systems. The flux qubits have an advantage over other types of superconducting qubits in the larger anharmonic energy level structure, i.e. the difference between adjacent transition frequencies is larger [25] and thus less leakage to higher energy-level states. Several schemes to implement local qubit operations and controllable couplings using microwaves for multi-flux-qubit systems have been proposed or/and realized. These schemes may be categorized into two groups: (i) directly coupling between qubits [26 30] and (ii) indirectly coupling through an intermediate coupler [18, 27, 31 33]. For example, a scheme to control the effective qubitqubit interaction for directly couping flux qubits with fixed qubit transition frequencies and large detuning between the neighboring qubits using time-dependent magnetic fluxes (or microwaves) was proposed in Ref. 28. While this approach is advantageous due to the resonant nature of the coupling, its disadvantage is that at least one of the qubits must be biased away from the coherence optimal point [4, 6, 9, 10]; this makes the qubit susceptible to low-frequency flux noise and results in a shorter coherence time. For the case of indirect coupling through an intermediate coupler, the coupling subcircuit or coupling nonlinear element can also be driven with microwaves [18, 27, 31 33]. These indirectly coupling schemes have the advantage of enabling the qubits to be operated at their optimal coherence points [4, 6, 9, 10]. However, in addition to the added circuit complexity, the effective couplings between two qubits in the indirectly coupling schemes are generally smaller so the two-qubit gating time is about $10-200 \mathrm{~ns}$, at least one or two order(s) of magnitude longer than that of directly coupling schemes. Schemes for microwave controllable coupling, which allow qubits to be operated at optimal bias points and without intermediate coupler subcircuit, exist [26 30]. But the effective qubit-qubit couplings in these schemes are usually even smaller than those of the indirectly coupling schemes. Smaller two-qubit coupling or equivalently longer two-qubit gating time will usually make the qubits suffer more decoherence effect during the gate operation, thus deteriorating the gate fidelity.

The fidelities of single-qubit and two-qubit quantum gate operations in a multi-qubit register for the present directly/indirectly coupling schemes via microwaves in the unitary case (without considering decoherence effect) even under the two-level qubit approximation are not perfect [18, 26 33]. The microwave pulses for single-qubit gate operations are generally obtained and applied under the approximation that the qubit with large detuning with its neighboring qubits is effectively decoupled with its neighbors even though there exist fixed two-qubit couplings between them. But this decoupling is only valid in the first order of a small parameter that is the ratio of the two-qubit coupling to the detuning. For two-qubit operations, the microwave pulses are commonly constructed and performed under the rotating wave approximation, the adiabatic approximation for the nonlinear coupler, or/and the approximation of neglecting other small residual two-qubit interactions. The 
qubit-decoupled approximation becomes excellent when the effective qubit-qubit coupling is very small; however, a large qubit-qubit interaction is favorable for two-qubit operations. Therefore, reaching a delicate balance between these two situations results in, for instance, single-qubit gate errors greater than $10^{-3}$ for practical experimental parameters even in the ideal, unitary case of simple two-level approximation for each flux qubit.

Quantum optimal control theory is a powerful tool that provides a variational framework for finding optimal control field profiles or sequences by maximizing a desired physical objective (or minimizing a physical cost function) within certain constraints [34 47]. In this paper, we apply the quantum optimal control theory to find the control pulse sequences of externally applied ac magnetic fluxes (fields) to implement fast and high-fidelity one-qubit and two-qubit gates on superconducting flux qubits. There have been optimal control studies of quantum gates for superconducting qubits, but all focusing on the gate operations of Cooper-pair-box charge qubit systems [38, 40, 42 44]. Our investigation here is, to our knowledge, the first optimal control study for the flux qubit systems. The flux qubits we consider have fixed direct qubit-qubit couplings and fixed transition frequencies but with large detuning between neighboring qubits, which ensures the qubits are effectively decoupled (to the first order) in the absence of time-dependent control fields or signals. Our optimal control scheme requires no additional bias or control lines beyond those used for the manipulation of individual qubits. In the absence of the time-dependent magnetic fluxes, the flux qubits in our scheme are biased at the coherence optimal points [4, 6, 9, 10] to reduce dephasing due to flux noise, i.e.,to have a longer coherence time. Furthermore, our optimal control scheme takes into account two-qubit interaction, qubit detuning and other time-dependent magnetic field induced residual single-qubit and two-qubit interactions when performing single-qubit and two-qubit gates. Thus the gate fidelities (errors) in the unitary (closed-system) case can be as high (low) as one wishes, limited only by the accuracy of the approximated two-level qubit Hamiltonian used and by the machine precision of the computation. Besides, the two-qubit CNOT gate operations of our scheme is about several folds to two orders of magnitude faster than the directly or/and indirectly microwave controllable coupling schemes [18, 26 33]. By considering leakage to higher-energy-level states, the gate errors for single-qubit gates using the optimal control pulse sequences obtained are in the order of $10^{-8}$ and are in the order of $10^{-6}$ for two-qubit CNOT gates. To take the effect of qubit decoherence into account, we model the qubit dynamics by a quantum master equation with experimentally available relaxation and dephasing rates. The gate errors in the presence of decoheence by considering the master equation are still in the order of $10^{-6}$ for single-qubit gates and in the order of $10^{-5}$ for two-qubit CNOT gates. These gate errors are still below the error threshold $10^{-4}\left(10^{-3}\right.$ in [48]; $10^{-2}$ if surface code error correction is used [49 51]) required for fault-tolerant quantum computation.

The paper is organized as follows. We first describe in Sec. [I] the Hamiltonian of two inductively coupled flux qubits with each individual qubit controlled by a time-dependent magnetic flux (field). The reduced Hamiltonian at the optimal bias point expressed in terms of the two-level qubit basis states is then obtained. In Sec. III, a brief description about the quantum optimal control theory for performing state-independent quantum gate operations is presented. The control field pulse sequences obtained by the optimal control theory and their corresponding gate errors and state evolutions are presented in Sec. IV] It is also shown that our optimal control scheme is notably robust against leakage to states outside the computational basis state space. The effect of qubit decoherence on gate errors is also discussed. A short conclusion with discussions of how to implement the optimal control 


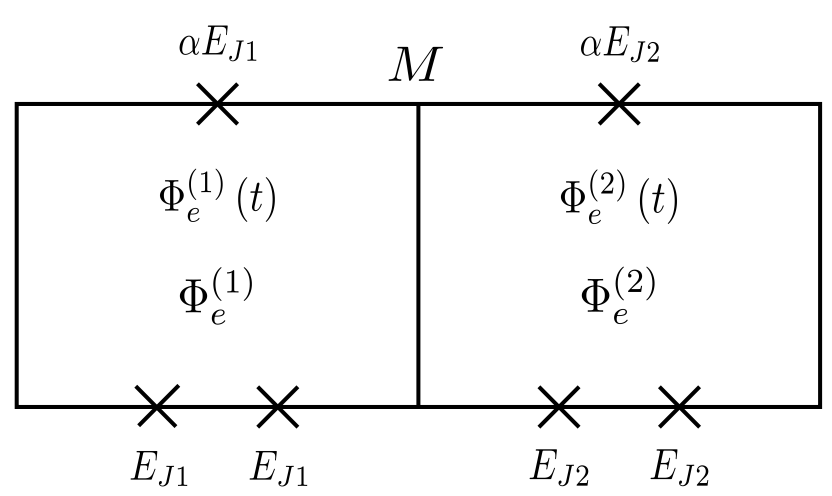

FIG. 1. (Color online) Schematic illustration of two inductively coupled superconducting flux qubits. The mutual inductance is $M$ and there are three junctions in each qubit loop. The external static and time-dependent magnetic fluxes through the $l$ th qubit are denoted as $\Phi_{e}^{(l)}$ and $\Phi_{e}^{(l)}(t)$, respectively.

pulses experimentally is given in Sec. $\mathrm{V}$.

\section{HAMILTONIAN OF COUPLED FLUX QUBITS}

The system we consider here is two flux qubits right next to each other and coupled by their mutual inductance $M$ [28, 52] as schematically illustrated in Fig. 1] Each qubit loop contains three Josephson junctions [5, 53], and one of them has an area $\alpha$ times smaller than that of the two identical junctions. The larger Josephson junctions in the $l$ th qubit loop have the Josephson energy $E_{J, l}$ and the corresponding critical current $I_{0}^{(l)} \equiv 2 \pi E_{J, l} / \Phi_{0}$, where $\Phi_{0}=h / 2 e$ is the flux quantum with the Planck constant $h$ and the elementary charge $e$. The capacitances in the $l$ th qubit loop satisfy the conditions $C_{1}^{(l)}=C_{2}^{(l)}=C_{J, l}$ and $C_{3}^{(l)}=\alpha C_{J, l}$, where $C_{1}^{(l)}=C_{2}^{(l)}$ are the capacitance of the larger Josephson junctions. The coupled flux qubit system considered here is exactly the same as that discussed in Ref. [28]. Fluxoid quantization around each qubit loop imposes a constraint on the phase drop across the three junctions [28]:

$$
\sum_{i} \varphi_{i}^{(l)}+2 \pi \frac{\Phi_{e}^{(l)}}{\Phi_{0}}+2 \pi \frac{\Phi_{e}^{(l)}(t)}{\Phi_{0}}=0
$$

where $\varphi_{i}^{(l)}$ is the gauge-invariant phase of each junction, $\Phi_{e}^{(l)}$ and $\Phi_{e}^{(l)}(t)$ are, respectively, the static $(\mathrm{dc})$ and time-dependent magnetic fluxes applied through the $l$ th qubit. Here the self-inductance is considered negligible as compared to the Josephson inductance. The requirement for our coupled-qubit system is to have suitable mutual inductance for qubits placed next to each other as illustrated in Fig. 1 and independent flux lines to enable control over each individual qubit with external dc bias magnetic fluxes and time-dependent control magnetic fluxes. The qubit-loop size for three-junction flux qubits could range experimentally from submicrometer to micrometer. As long as the design of the qubit loops fulfills the above requirement, the actual size of each qubit loop or the distance between the qubits can have some flexibility. 
The total Hamiltonian of the two coupled three-junction flux qubits reads [28, 53]:

$$
\begin{aligned}
H=M I_{1} I_{2}+\sum_{l} & \left\{\frac{P_{P, l}^{2}}{2 M_{P, l}}+\frac{P_{Q, l}^{2}}{2 M_{Q, l}}\right. \\
+ & 2 \pi \dot{f}_{c}^{(l)}(t)\left(\frac{\alpha}{1+2 \alpha}\right) P_{P, l}+2 E_{J, l}\left(1-\cos \varphi_{Q}^{(l)} \cos \varphi_{P}^{(l)}\right) \\
& \left.+\alpha E_{J, l}\left[1-\cos \left(2 \varphi_{P}^{(l)}+2 \pi f_{l}+2 \pi f_{c}^{(l)}(t)\right)\right]\right\},
\end{aligned}
$$

with the redefined phases $\varphi_{P}^{(l)}=\left(\varphi_{1}^{(l)}+\varphi_{2}^{(l)}\right) / 2, \varphi_{Q}^{(l)}=\left(\varphi_{1}^{(l)}-\varphi_{2}^{(l)}\right) / 2$, the reduced dc bias magnetic flux $f_{l}=\Phi_{e}^{(l)} / \Phi_{0}$, and the reduced time-dependent control magnetic flux $f_{c}^{(l)}(t)=$ $\Phi_{e}^{(l)}(t) / \Phi_{0}$. The effective masses are $M_{Q, l}=2\left(\Phi_{0} / 2 \pi\right)^{2} C_{J, l}$ and $M_{P, l}=(1+2 \alpha) M_{Q, l}$, which correspond to the effective momenta $P_{Q, l}=-\imath \hbar\left(\partial / \partial \varphi_{Q}^{(l)}\right)$ and $P_{P, l}=-\imath \hbar\left(\partial / \partial \varphi_{P}^{(l)}\right)$. The persistence current in the $l$ th qubit loop is

$$
\begin{aligned}
& I_{l}=\frac{\alpha I_{0}^{(l)}}{1+2 \alpha}\left[\sin \left(\varphi_{P}^{(l)}+\varphi_{Q}^{(l)}\right)+\sin \left(\varphi_{P}^{(l)}-\varphi_{Q}^{(l)}\right)\right. \\
& \left.+\sin \left(2 \varphi_{P}^{(l)}+2 \pi f_{l}+2 \pi f_{c}^{(l)}(t)\right)\right] .
\end{aligned}
$$

Here the self-interaction terms of the external time-dependent control magnetic fields $\sum_{l=1}^{2}(\alpha / 2) C_{J, l}\left[\dot{\Phi}_{e}^{(l)}(t)\right]^{2}$ is neglected since they vary only the global phase of the state vector and do not affect the control of the coupled flux qubits. To simulate the dynamics of the coupled flux-qubit system with Hamiltonian Eq. (2), the parameter values of the mutual conductance $M$ as well as the Josephson energies of the junctions $E_{J, l}$, the capacitance of the larger Josephson junctions $C_{J, l}$ and the area ratio $\alpha$ of the smaller junction to one of the larger junctions in each qubit loop are required. These parameter values can be obtained through the experimental measurement and characterization of the system. The actual values of these parameters we choose for our simulations are given in Sec. IV] They are all experimentally available or realistic values.

We will keep the time-dependent control amplitudes small such that the reduced timedependent magnetic flux satisfies $\left|f_{c}^{(l)}(t)\right|=\left|\Phi_{e}^{(l)}(t) / \Phi_{0}\right| \lesssim 10^{-3}$. Small time-dependent control amplitude allows the approximation of $\sin \left[2 \pi f_{c}^{(l)}(t)\right] \sim 2 \pi f_{c}^{(l)}(t)$ and $\cos \left[2 \pi f_{c}^{(l)}(t)\right] \sim 1$ when expanding the last term of the Hamiltonian of Eq. (2). As a result, the time-dependent Hamiltonian becomes linear in $f_{c}^{(l)}(t)$ or $f_{c}^{(1)}(t) f_{c}^{(2)}(t)$, which can be readily incorporated with the Krotov quantum optimal control method that we will employ later. This weakamplitude approximation of the control fields also keeps the qubit not deviating much from the dc bias point that is set to be the optimal coherence point in our case. Moreover, this weak-amplitude approximation helps reduce unwanted possible excitations to the higherenergy-level states outside the computational state space when we make two-level (qubit) approximation discussed later.

Making this weak-amplitude approximation, we obtain from Eq. (2) the Hamiltonian similar to that in Ref. [28] as

$$
H=\sum_{l=1}^{2}\left(H_{l}+H_{D}^{(l)}\right)+\sum_{l \neq m=1}^{2} H_{l m}+H_{C}+H_{A} .
$$


The first term $H_{l}$ is the single-qubit Hamiltonian and reads:

$$
\begin{aligned}
H_{l}= & \frac{P_{P, l}^{2}}{2 M_{P, l}}+\frac{P_{Q, l}^{2}}{2 M_{Q, l}}+2 E_{J, l}\left(1-\cos \varphi_{Q}^{(l)} \cos \varphi_{P}^{(l)}\right) \\
& +\alpha E_{J, l}\left[1-\cos \left(2 \varphi_{P}^{(l)}+2 \pi f_{l}\right)\right] .
\end{aligned}
$$

The Hamiltonian $H_{D}^{(l)}$ plays the role of a driving Hamiltonian representing the interaction between the $l$ th qubit and its time-dependent magnetic field. It takes the form

$$
H_{D}^{(l)}=2 \pi \alpha\left[f_{c}^{(l)}(t) E_{J, l} \sin \left(2 \varphi_{P}^{(l)}+2 \pi f_{l}\right)+\dot{f}_{c}^{(l)}(t) \frac{P_{P, l}}{1+2 \alpha}\right]
$$

The last three terms in Eq. (44) come from the inductive coupling between the two flux qubits. The Hamiltonian $H_{l m}$ describes the qubit-qubit interaction controlled by one of the time-dependent magnetic flux $\left[f_{c}^{(1)}(t)\right.$ or $\left.f_{c}^{(2)}(t)\right]$ and is written as

$$
H_{l m}=-2 \pi M\left(\frac{\alpha}{1+2 \alpha}\right) I^{(l)} I_{0}^{(m)} f_{c}^{(m)}(t) \cos \left(2 \varphi_{P}^{(m)}+2 \pi f_{m}\right)
$$

where

$$
\begin{aligned}
I^{(l)}=\frac{\alpha I_{0}^{(l)}}{1+2 \alpha}[ & \sin \left(\varphi_{P}^{(l)}+\varphi_{Q}^{(l)}\right)+\sin \left(\varphi_{P}^{(l)}-\varphi_{Q}^{(l)}\right) \\
& \left.-\sin \left(2 \varphi_{P}^{(l)}+2 \pi f_{l}\right)\right]
\end{aligned}
$$

is the loop current of the $l$ th qubit when no time-dependent magnetic fluxes are applied [cf., Eq. (3)]. The qubit-qubit interaction $H_{C}$ controlled by two simultaneously applied time-dependent magnetic fluxes through, respectively, the two qubits is

$$
H_{C}=M\left(\frac{2 \pi \alpha}{1+2 \alpha}\right)^{2} \prod_{l=1}^{2} I_{0}^{(l)} f_{c}^{(l)}(t) \cos \left(2 \varphi_{P}^{(l)}+2 \pi f_{l}\right)
$$

We still keep this term, although it is much smaller than the term controlled by a single time-dependent magnetic flux. The Hamiltonian

$$
H_{A}=M I^{(1)} I^{(2)}
$$

describes an always-on interaction between the two flux qubits without time-dependent magnetic fluxes being applied, where $I^{(l)}$ is defined in Eq. (8).

The Hamiltonian, Eq. (4), in the two-qubit computational state basis $\left\{\left|g_{1}\right\rangle,\left|e_{1}\right\rangle\right\} \otimes$ $\left\{\left|g_{2}\right\rangle,\left|e_{2}\right\rangle\right\}$, where $\left|g_{l}\right\rangle$ and $\left|e_{l}\right\rangle$ are the lowest two energy-level states of $H_{l}$ of Eq. (5), 
becomes

$$
\begin{aligned}
\frac{H}{\hbar}= & \sum_{l=1}^{2}\left[\frac{\omega_{l}}{2} \sigma_{z}^{(l)}+\kappa_{1}^{(l)}(t) \sigma_{z}^{(l)}+\kappa_{2}^{(l)}(t) \sigma_{x}^{(l)}\right] \\
& -\sum_{l \neq m=1}^{2}\left[\chi_{1}^{(l m)}(t) \sigma_{z}^{(l)}+\chi_{2}^{(l m)}(t) \sigma_{x}^{(l)}\right] \\
& -\sum_{l \neq m=1}^{2}\left[\Xi_{11}^{(l m)}(t) \sigma_{z}^{(l)} \sigma_{z}^{(m)}+\Xi_{22}^{(l m)}(t) \sigma_{x}^{(l)} \sigma_{x}^{(m)}\right. \\
& \left.\quad+\Xi_{12}^{(l m)}(t) \sigma_{z}^{(l)} \sigma_{x}^{(m)}+\Xi_{21}^{(l m)}(t) \sigma_{x}^{(l)} \sigma_{z}^{(m)}\right] \\
& +\Theta_{11}(t) \sigma_{z}^{(1)} \sigma_{z}^{(2)}+\Theta_{22}(t) \sigma_{x}^{(1)} \sigma_{x}^{(2)}+\Theta_{12}(t) \sigma_{z}^{(1)} \sigma_{x}^{(2)}+\Theta_{21}(t) \sigma_{x}^{(1)} \sigma_{z}^{(2)} \\
& +\Lambda_{11} \sigma_{z}^{(1)} \sigma_{z}^{(2)}+\Lambda_{22} \sigma_{x}^{(1)} \sigma_{x}^{(2)}+\Lambda_{12} \sigma_{z}^{(1)} \sigma_{x}^{(2)}+\Lambda_{21} \sigma_{x}^{(1)} \sigma_{z}^{(2)}
\end{aligned}
$$

where the single-qubit transition frequencies $\omega_{l}$ and the driving Hamiltonian amplitudes $\kappa_{1}^{(l)}(t)$ and $\kappa_{2}^{(l)}(t)$ are

$$
\begin{gathered}
\omega_{l}=\frac{1}{\hbar}\left(\left\langle e_{l}\left|H_{l}\right| e_{l}\right\rangle-\left\langle g_{l}\left|H_{l}\right| g_{l}\right\rangle\right), \\
\kappa_{1}^{(l)}(t)=\frac{1}{2 \hbar}\left(\left\langle e_{l}\left|H_{D}^{(l)}\right| e_{l}\right\rangle-\left\langle g\left|H_{D}^{(l)}\right| g_{l}\right\rangle\right), \\
\kappa_{2}^{(l)}(t)=\frac{1}{\hbar}\left\langle e_{l}\left|H_{D}^{(l)}\right| g_{l}\right\rangle,
\end{gathered}
$$

respectively, the other time-dependent controllable interaction strengths, $\chi_{1}^{(l m)}(t), \chi_{2}^{(l m)}(t)$, $\Xi_{i j}^{(l m)}(t)$ and $\Theta_{i j}(t)$, coming from qubit-qubit inductive interaction are

$$
\begin{gathered}
\chi_{1}^{(l m)}(t)=\frac{2 \pi}{\hbar} \beta_{M} f_{c}^{(l)}(t) \Omega_{1}^{(l)} \Delta^{(m)}, \\
\chi_{2}^{(l m)}(t)=\frac{2 \pi}{\hbar} \beta_{M} f_{c}^{(l)}(t) \Omega_{2}^{(l)} \Delta^{(m)}, \\
\Xi_{i j}^{(l m)}(t)=\frac{2 \pi}{\hbar} \beta_{M} f_{c}^{(l)}(t) \Omega_{i}^{(l)} \lambda_{j}^{(m)}, \\
\Theta_{i j}(t)=\frac{(2 \pi)^{2}}{\hbar} \beta_{M} f_{c}^{(1)}(t) f_{c}^{(2)}(t) \Omega_{i}^{(1)} \Omega_{j}^{(2)},
\end{gathered}
$$

respectively, and the static fixed qubit-qubit interaction strengths $\Lambda_{i j}$ are

$$
\Lambda_{i j}=\frac{\beta_{M}}{\hbar} \lambda_{i}^{(1)} \lambda_{j}^{(2)}
$$

The relevant parameters in Eqs. (15)-(19) are $\beta_{M}=M I_{0}^{(1)} I_{0}^{(2)}$ which is the mutual inductive energy with respect to the critical current $I_{0}^{(l)}=2 \pi E_{J, l} / \Phi_{0}$ of the larger Josephson junctions in each qubit loop,

$$
\lambda_{1}^{(l)}=\frac{1}{2}\left(\left\langle e_{l}\left|\frac{I^{(l)}}{I_{0}^{(l)}}\right| e_{l}\right\rangle-\left\langle g_{l}\left|\frac{I^{(l)}}{I_{0}^{(l)}}\right| g_{l}\right\rangle\right),
$$




$$
\begin{gathered}
\lambda_{2}^{(l)}=\left\langle e_{l}\left|\frac{I^{(l)}}{I_{0}^{(l)}}\right| g_{l}\right\rangle, \\
\Omega_{1}^{(l)}=\frac{1}{2}\left[\left\langle e_{l}\left|\left(\Upsilon^{(l)}-\frac{I^{(l)}}{I_{0}^{(l)}}\right)\right| e_{l}\right\rangle-\left\langle e_{l}\left|\left(\Upsilon^{(l)}-\frac{I^{(l)}}{I_{0}^{(l)}}\right)\right| e_{l}\right\rangle\right], \\
\Omega_{2}^{(l)}=\left\langle e_{l}\left|\left(\Upsilon^{(l)}-\frac{I^{(l)}}{I_{0}^{(l)}}\right)\right| g_{l}\right\rangle,
\end{gathered}
$$

and

$$
\Delta^{(l)}=\frac{1}{2}\left[\left\langle e_{l}\left|\Upsilon^{(l)}\right| e_{l}\right\rangle+\left\langle g_{l}\left|\Upsilon^{(l)}\right| g_{l}\right\rangle\right]
$$

where the operator $\Upsilon^{(l)}$ is defined as

$$
\Upsilon^{(l)} \equiv \frac{\alpha}{1+2 \alpha} \cos \left(2 \varphi_{P}^{(l)}+2 \pi f_{l}\right)+\frac{I^{(l)}}{I_{0}^{(l)}}
$$

The Hamiltonian Eq. (11) with parameters defined in Eqs. (12)-(25) is valid for weak timedependent magnetic fluxes (fields) and arbitrary static bias magnetic fluxes. If we take the time dependence of the control magnetic flux to be sinusoidal, i.e., $e^{ \pm i \omega t}$, and making the relevant rotating wave approximation, we can revert to the Hamiltonian of Ref. [28]. Different from Ref. [28], our control scheme, however, does not require qubits being biased away from the optimal coherence points. In contrast, we set the reduced static or dc bias magnetic fluxes to be $f_{1}=f_{2}=0.5$ so that the qubits are at the optimal coherence points and are thus insensitive to low-frequency flux noise in the first order. At the optimal coherence points of $f_{1}=f_{2}=0.5$, Hamiltonian $H_{l}$ of Eq. (5) is invariant under the parity transformation of $\varphi_{P}^{(l)}$, i.e., $H_{l}\left(-\varphi_{P}^{(l)}\right)=H_{l}\left(\varphi_{P}^{(l)}\right)$. Thus its eigenstates have definite parities and the lowest two energy-level eigenstates have the opposite parities [54, 55]. Since the Hamiltonian of Eq. (6) and the loop currents of Eq. (8) are odd functions of $\varphi_{P}^{(l)}$ and the operators $\Upsilon^{(l)}-\left(I^{(l)} / I_{0}^{(l)}\right)$ from Eq. (25) are even functions of $\varphi_{P}^{(l)}$ at $f_{1}=f_{2}=0.5$, the parameters $\kappa_{1}^{(l)}(t), \lambda_{1}^{(l)}$ and $\Omega_{2}^{(l)}$ defined in Eqs. (13), (20) and (23), respectively, vanish due to the parity symmetry consideration. As a consequence, most parameters in Eqs. (15)-(19) vanish, and Eq (11) at the optimal bias points simplifies to

$$
\begin{aligned}
\frac{H}{\hbar}= & \sum_{l=1}^{2}\left[\frac{\omega_{l}}{2} \sigma_{z}^{(l)}+\kappa_{2}^{(l)}(t) \sigma_{x}^{(l)}\right]+\Lambda_{22} \sigma_{x}^{(1)} \sigma_{x}^{(2)} \\
& -\sum_{l \neq m=1}^{2}\left[\chi_{1}^{(l m)}(t) \sigma_{z}^{(l)}+\Xi_{12}^{(l m)}(t) \sigma_{z}^{(l)} \sigma_{x}^{(m)}\right]+\Theta_{11}(t) \sigma_{z}^{(1)} \sigma_{z}^{(2)} .
\end{aligned}
$$

We will use Eq. (26) combining with quantum optimal control theory to find control magnetic field pulses $f_{c}^{(l)}(t)$ for the implementation of high-fidelity one- and two-qubit gates. 


\section{KROTOV QUANTUM OPTIMAL CONTROL METHOD}

Quantum optimal control theory enables us to realize accurate state-independent quantum gates by selecting optimal pulse shapes (arbitrarily shaped pulses and duration; or continuous dynamical modulation) for the external control within experimental capabilities [37, 38, 40, 42 47]. To perform state-independent optimal control, the equation of motion for the time evolution operator (or propagator) $U(t)$ is needed:

$$
\imath \hbar \frac{\partial}{\partial t} U(t)=H[t, \epsilon(t)] U(t)
$$

where $H$ is the system Hamiltonian and $\epsilon(t)$ is a time-dependent control field. We choose the trace distance between the desired target gate operation $O$ and the actual (could be nonunitary) propagator $U(T)$ at the final operation time $T$ to characterize the gate error:

$$
\eta=\frac{1}{2 N} \operatorname{Tr}\left\{[O-U(T)]^{\dagger}[O-U(T)]\right\},
$$

where $N$ is the dimension of the matrix $U(t)$. If the actual time evolution operator $U(T)$ is equal to the target gate operation $O$, then $\eta=1-\mathcal{F}=0$ with $\mathcal{F}$ denoting the gate fidelity. Sometimes, one is also interested in calculating the errors of the operations (operators or matrices) in a subspace of a unitary matrix. When projection is made to the subspace, the projected matrix in the subspace is in general no longer unitary. For instance, when the evolution operator of the multi-level coupled flux-qubit system $U(T)$ for the multi-level Hamiltonian, Eq. (2), is projected into the approximated two-level qubit computational state subspace, the projected time evolution operator is no longer unitary. In this case, Eq. (28) with slight modification [see Eq. (30)] is still an appropriate measure for calculating the gate error.

In realistic control problems, it is desirable that the optimal control sequence can provide highest quality (fidelity) with minimum energy consumption. Therefore, we define the cost function for our optimal control problem as

$$
J=\eta+\int_{0}^{T} \frac{\lambda}{S(t)}\left[\epsilon(t)-\epsilon_{\mathrm{ref}}(t)\right]^{2} d t,
$$

where $\eta$ is defined in Eq. (28), $S(t)$, a positive shape function, and $\lambda$, a weight, can be adjusted and chosen empirically [35, 36]. Here, the reference field $\epsilon_{\text {ref }}(t)$ is chosen to be the control sequence in the previous iteration, i.e., $\epsilon_{\text {ref }}(t)=\epsilon^{(i)}(t)$ such that the control field energy constraint in the cost function $J$ of Eq. (29) has the physical interpretation that the change of the control pulse energy in each iteration is limited [35, 36]. When the iterative procedure approaches the optimal solution, the change in the control field is minimal or vanishing. Therefore, this choice of the reference field $\epsilon_{\mathrm{ref}}(t)$ ensures that the iterative method is found to reduce the total objective $J$ of Eq. (29) by reducing the gate error $\eta$ rather than the total control pulse energy. With Eqs. (27) and (29) and the initial propagator $U(0)=I$, where $I$ is the identity operator, one can then investigate the state-independent quantum gate optimal control problem.

We will use the Krotov iterative method for the quantum gate optimal control 35, 39, 41, 46, 47, 56]. The Krotov method has several appealing advantages [35, 39, 41] over the standard gradient optimization methods: (a) monotonic increase of the objective with iteration number, (b) no requirement for a line search, and (c) macrosteps at each iteration. The optimal algorithm following the Krotov method [56] can be found in Refs. 35, 36, 41, and 46. 


\section{QUANTUM GATE OPERATIONS VIA QUANTUM OPTIMAL CONTROL THEORY}

We use the reduced Hamiltonian, Eq. (26), with two lowest energy-level states for each qubit to obtain control sequences for single-qubit $X$ and $Z$ gate operations and two-qubit CNOT gate operations by optimizing the cost function $J$, Eq. (29), using the Krotov iterative method. Simulations on the two-qubit Hamiltonian, Eq. (2), without making the weakamplitude approximation and with the lowest five energy states for each qubit, using the optimal control field sequences found via Eq. (26) will also be performed for error comparison. This allows us to test how well the optimal control pulses obtained by the weak-amplitude approximation perform, and to determine how severe the leakage to the higher-energy-level states is. The target gate operation $O$ is defined in the two-qubit computational state basis. The evolution operator or propagator $U(T)$ results from Eq. (2) using the optimal control pulse sequences $f_{c}^{(1)}(t)=\Phi_{e}^{(1)}(t) / \Phi_{0}$ and $f_{c}^{(2)}(t)=\Phi_{e}^{(2)}(t) / \Phi_{0}$ obtained from Eq. (26) is in a larger multi-level-state space. We thus define a projection operator $P$ to project $U(T)$ onto the subspace subtended by the computational basis states, and compare the target operator $O$ with the resultant propagator $P U(T)$ which is in general no longer unitary. The error in this case is defined from Eq. (28) as

$$
\eta_{P}=(1 / 2 N) \operatorname{Tr}\left\{[O-P U(t)]^{\dagger}[O-P U(t)]\right\},
$$

where $N$ is the dimension of the $O$ matrix or $P U(t)$ matrix.

We choose the parameters in the two-qubit Hamiltonian with experimentally available or realistic values [5]. The Josephson energy of the larger junctions in the qubit loops is chosen to be $E_{J, 1} / \hbar=2 E_{J, 2} /(5 \hbar)=E_{J} / \hbar=2 \pi \times 248.72 \mathrm{GHz}$ and the charging energy $E_{C, l}=e^{2} / 2 C_{J, l}$ is chosen to be $E_{J, l} / E_{C, l}=35$. The capacitance of the smaller Josephson junction is $C_{3, l}=\alpha C_{J, l}$ with the ratio $\alpha=0.8$. To fit the value of the coupling strength $\Lambda_{22}=2 \pi \times 0.4 \mathrm{GHz}$ reported in the experiment of Ref. 57, we take the mutual inductance $M=1 \mathrm{pH}$ and obtain $\beta_{M}=3.75 \times 10^{-3} E_{J}$. Then other parameters are determined by the calculations through the Hamiltonian, Eq. (2). We then obtain the qubit transition energies $\omega_{1}=2 \pi \times 3.30 \mathrm{GHz}$ and $\omega_{2}=2 \pi \times 8.24 \mathrm{GHz}$, and the single-qubit driving amplitudes $\kappa_{2}^{(1)}=-2 \pi f_{c}^{(1)}(t) \times 1.02 \times 10^{3} \mathrm{GHz}$ and $\kappa_{2}^{(2)}=-2 \pi f_{c}^{(2)}(t) \times 2.57 \times 10^{3} \mathrm{GHz}$. The time-dependent coefficients of the last three coupling terms in Eq. (26) are also obtained to be $\chi_{1}^{(l m)}=2 \pi f_{c}^{(l)}(t) \times 4.4 \times 10^{-3} \mathrm{GHz}, \Xi_{12}^{(l m)}=2 \pi f_{c}^{(l)}(t) \times 8.22 \times 10^{-2} \mathrm{GHz}$, and $\Theta_{11}=2 \pi f_{c}^{(1)}(t) f_{c}^{(2)}(t) \times 1.66 \times 10^{-2} \mathrm{GHz}$. We note here that the dynamics and also the optimization results that will be presented later depend on the values of the microscopic parameters. These parameter values can be obtained through the experimental measurement and characterization of the system but always come with some error bars. Thus if the actual system is, for example, slightly away from the assumed control point (a set of values for the system parameters) chosen for the quantum optimal control theory, then the optimal control pulse sequences sent to the experiment may result in gate fidelities not close to optimal. This issue of imprecise knowledge of the system parameters seems to hinder the practical use and experimental applicability of the quantum optimal control theory. Fortunately, there may be ways around, for example, using the adaptive model-free closed-loop feedback control method. We postpone the discussions regarding this until Sec. V.

In the following, we first present our optimal control results for single-qubit and twoqubit gates in the unitary case together with brief descriptions of corresponding conventional 


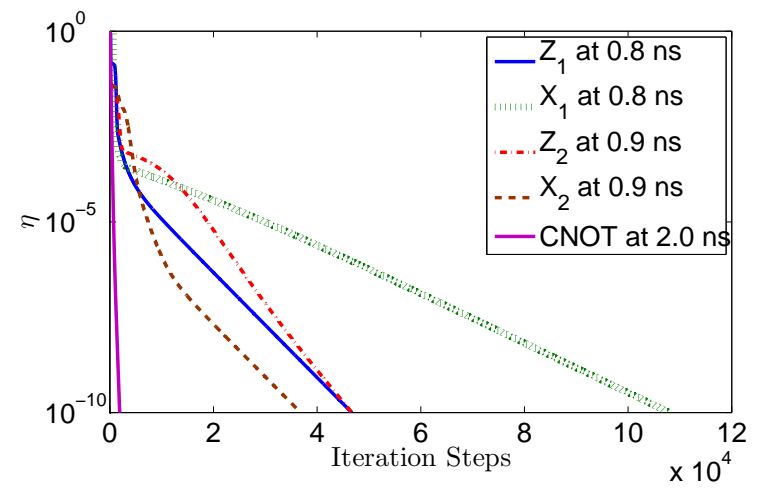

FIG. 2. (Color online) Gate errors $\eta$ as a function of the number of iterations. The gate errors calculated using Hamiltonian Eq. (31) decrease as the number of iterations increases. The stopping iteration criteria of error is set to be $\eta<10^{-10}$. The single-qubit gates of the first qubit, the singlequbit gates of the second qubit and the two-qubit CNOT gate are achieved at $T=0.8 \mathrm{~ns}, T=0.9$ ns and $T=2.0 \mathrm{~ns}$, respectively.

approaches for comparison. The results considering the effect of leakage to higher-energylevel states and the effect of qubit decoherence are presented subsequently.

\section{A. Single-qubit gate: unitary case}

Conventional approach - In most of the studies for the directly coupling schemes, the Hamiltonian of two inductively coupled flux qubits at the optimal bias point contains only the first line of Eq. (26):

$$
\frac{H}{\hbar}=\sum_{l=1}^{2}\left[\frac{\omega_{l}}{2} \sigma_{z}^{(l)}+\kappa_{2}^{(l)}(t) \sigma_{x}^{(l)}\right]+\Lambda_{22} \sigma_{x}^{(1)} \sigma_{x}^{(2)} .
$$

This is because the coefficients of the last three time-dependent terms of Eq. (26) are several orders of magnitude smaller than the qubit transition frequencies $\omega_{l}$, the single-qubit driving amplitudes $\kappa_{2}^{(l)}$ and the fixed static qubit-qubit interaction strength $\Lambda_{22}$. Thus neglecting these coefficients does not introduce appreciable error. Furthermore, since the strength $\Lambda_{22}$ of the off-diagonal coupling $\sigma_{x}^{(1)} \sigma_{x}^{(2)}$ in the presence of large qubit detuning $\left|\omega_{1}-\omega_{2}\right|$ affects the qubit dynamics only in second order in the parameter $\left(\Lambda_{22} /\left|\omega_{1}-\omega_{2}\right|\right)$, the constant coupling $\Lambda_{22}$ term is often neglected at the optimal bias point provided that $\left(\Lambda_{22} /\left|\omega_{1}-\omega_{2}\right|\right)$ is rather small. In this case, the qubits are regarded to be effectively decoupled. Thus the single-qubit gate, say, $X_{1}$ gate (rotation of angle $\pi$ around $x$-axis on qubit 1 ), can be achieved by simply driving the magnetic flux through the first qubit with microwave resonance with the transition frequency of the first qubit, i.e., $\kappa_{2}^{(1)}=\tilde{\kappa}_{2}^{(1)} \cos \left(\omega_{1} t\right)$, while keeping the timedependent flux through the second qubit off, i.e., $\kappa_{2}^{(2)}=0$. When the driving field strength $\tilde{\kappa}_{2}^{(1)}$ is much smaller than the transition frequencies $\omega_{l}$ and qubit detuning $\left|\omega_{1}-\omega_{2}\right|$ in which the rotating-wave approximation can be made, Eq. (31) in the frame rotating with the driving frequency $\omega_{1}$ becomes

$$
\frac{H_{\mathrm{rot}}}{\hbar}=\frac{\tilde{\kappa}_{2}^{(1)}}{2} \sigma_{x}^{(1)}+\frac{\omega_{2}}{2} \sigma_{z}^{(2)} .
$$


(a)

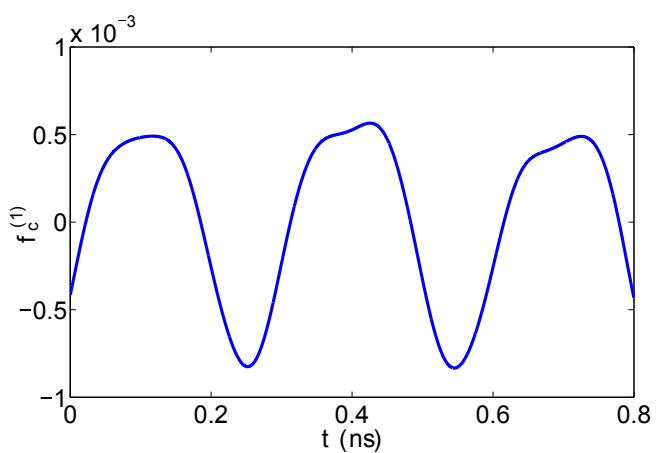

(c)

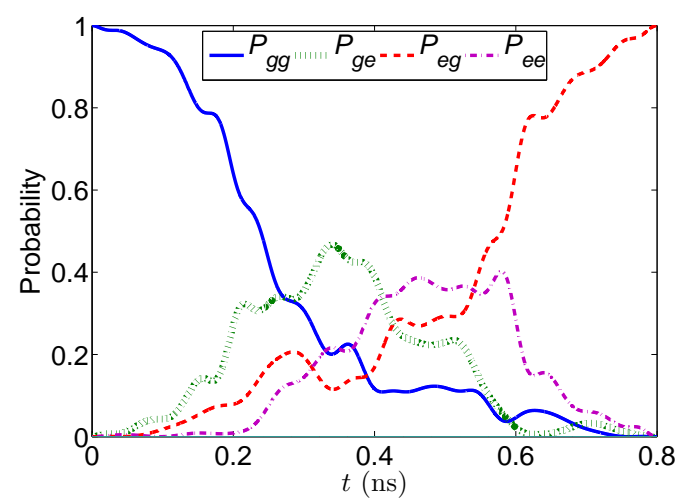

(e)

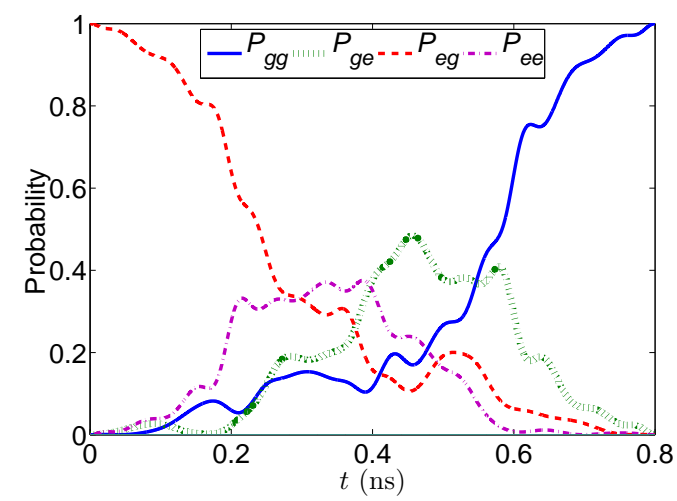

(b)

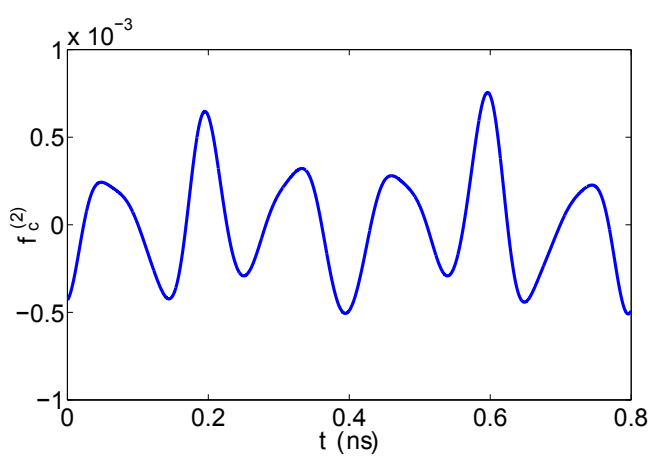

(d)

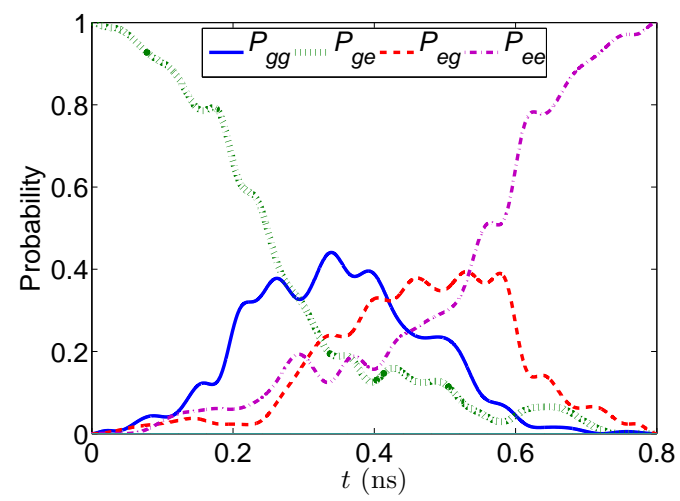

(f)

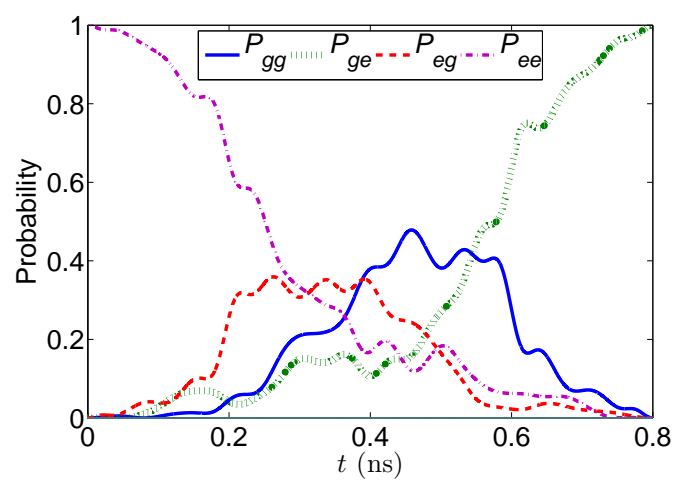

FIG. 3. (Color online) Optimal control sequences and state probability evolutions for a $X_{1}$ gate with operation time of $T=0.8$ ns. The optimal control pulse sequences of the reduced magnetic fluxes (a) $f_{c}^{(1)}(t)$ and (b) $f_{c}^{(2)}(t)$ are obtained via the Hamiltonian, Eq. (26). The time evolutions of the two-qubit computational-state probabilities calculated via the Hamiltonian, Eq. (2), with the lowest five energy states per qubit using the optimal control sequences shown in (a) and (b) are plotted in (c)-(f) with each two-qubit computational state as an initial state. The two-qubit computational basis states are $|g g\rangle,|g e\rangle,|e g\rangle$ and $|e e\rangle$. 
Performing single-qubit $X_{1}$-gate on qubit 1 will also demand qubit 2 to return to its original state up to a global phase at the end of the $X_{1}$ operation, i.e., an identity gate on qubit 2 . In this rotating frame, qubit 2 takes $T=2 n \pi / \omega_{2}$ to complete an identity gate up to a global phase with $n$ being an integer number. Taking this time to be the time to complete a $\pi$-pulse on qubit 1 yields the single-qubit driving strength or the Rabi frequency to be $\tilde{\kappa}_{2}^{(1)}=\omega_{2} / 2 n$. By requiring the reduced time-dependent magnetic flux $\left|f_{c}^{(1)}(t)\right|=\left|\Phi_{e}^{(1)}(t) / \Phi_{0}\right| \lesssim 10^{-3}$ such that the weak-amplitude approximation to obtain Eq. (4) is valid, one obtains the minimum integer number $n$ to be 7 . This then leads to $f_{c}^{(1)}(t) \approx 5.77 \times 10^{-4} \cos \left(\omega_{1} t\right)$ and the operation time of $X_{1}$ gate $T=14 \pi / \omega_{2} \approx 0.85$ ns. Plugging these numbers into Eq. (26) and Eq. (2) to simulate an $X_{1}$ gate gives an error of about $\eta \approx \eta_{P} \sim 3.3 \times 10^{-3}$. This result indicates that the dominant source of error is mainly due to the constant qubit-qubit interaction term of $\Lambda_{22}$ and does not come from the leakage to higher-energy-level states [58]. Indeed, for the experimental parameters used here, the ratio of $\left(\Lambda_{22} /\left|\omega_{1}-\omega_{2}\right|\right)=0.4 / 4.96 \approx 0.08$, and taking the second order correction gives an estimated error also in the order of $10^{-3}$. This is about the best one can do in a unitary case if neglecting the constant qubit-qubit interaction $\Lambda_{22}$ term. How to include the qubit-qubit interaction term and perform a much more accurate single-qubit gate is not intuitively obvious.

Optimal control approach - Here, we apply the Krotov iterative method to obtain the optimal control sequences for high-fidelity (low-error) single-qubit and two-qubit gates taking all the static and time-dependent single-qubit and two-qubit terms or interactions into account. Usually, stronger strengths of the control fields result in shorter gate operation times. In our case, the gate operation times are, however, chosen such that the reduced time-dependent magnetic fluxes satisfying the weak-amplitude approximation of $\left|f_{c}^{(l)}(t)\right|=\left|\Phi_{e}^{(l)}(t) / \Phi_{0}\right| \lesssim 10^{-3}$. We find that the high-fidelity single-qubit gates on qubit 1 can be achieved at a gating time of $T=0.8 \mathrm{~ns}$, and the gating time is $T=0.9 \mathrm{~ns}$ for qubit 2 . The two-qubit gates will be discussed in the next subsection. As shown in Fig. 2, the errors $\eta$ of $Z_{1}, X_{1}, Z_{2}$ and $X_{2}$ gates calculated using the reduced Hamiltonian, Eq. (26), decrease with the number of iterations. We note here that for simplicity, we let the positive shape function $S(t)$ in Eq. (29) be a constant. In addition, the time-dependent control field $\epsilon(t)$ in Eq. (29) in our case becomes the reduced time-dependent magnetic fluxes $\left|f_{c}^{(l)}(t)\right|=\left|\Phi_{e}^{(l)}(t) / \Phi_{0}\right|$ that are dimensionless and required to be smaller than $10^{-3}$, and thus in our calculations we choose $S(t) / \lambda=10^{-10}(\mathrm{GHz})^{-1}$ in order to make sure that the iterations converge to the optimal results monotonically. In principle, the gate error for a closed, unitary system can, via the optimal control theory, be as low as one wishes, limited only by the accuracy of the two-level-approximation Hamiltonian, Eq. (26), and by the machine precision of the computation. Considering the accuracy of the two-level-approximation Hamiltonian used, we set the stopping criteria of error to be $10^{-10}$, and thus the iterations are terminated when error $\eta<10^{-10}$. A typical set of the control sequences of the reduced time-dependent magnetic fluxes $f_{c}^{(1)}(t)$ and $f_{c}^{(2)}(t)$ for the high-fidelity $X_{1}$ gate is shown in Figs. $3[(a)$ and $3[(\mathrm{~b})$.

\section{B. Two-qubits gate: unitary case}

Conventional approach - Many schemes using microwaves have been put forward 18, 26 33. to realize an effective controllable qubit-qubit interaction for Hamiltonian (31) or (26). However, in most of the schemes, two neighboring qubits are considered effectively decoupled (as described earlier) when no time-dependent microwave field is applied. This 
(a)

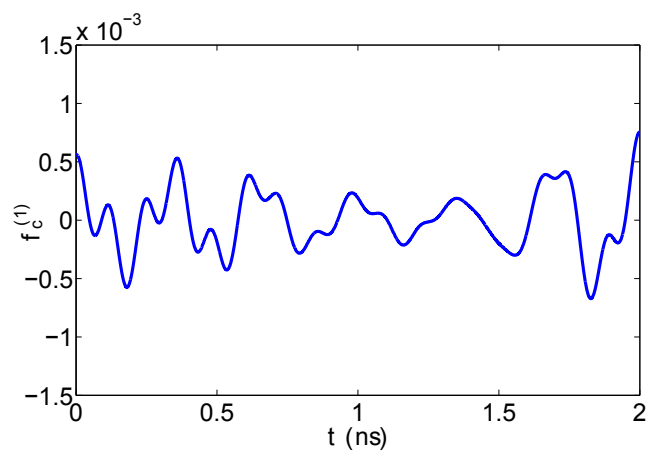

(c)

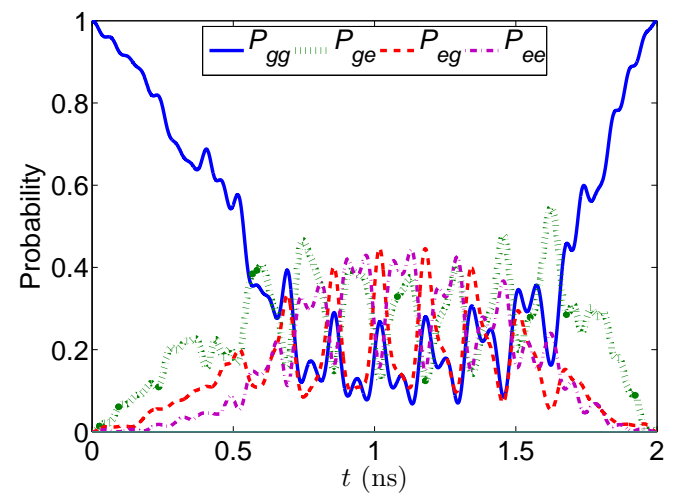

(e)

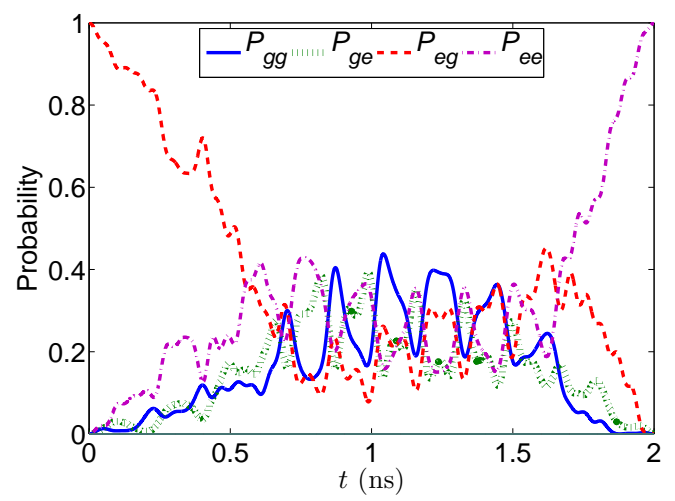

(b)

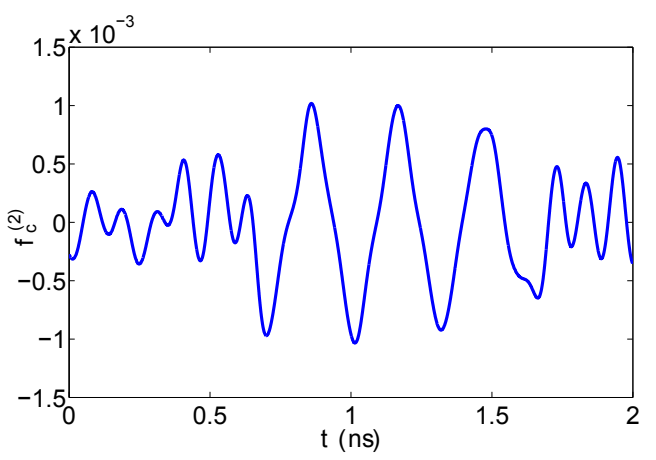

(d)

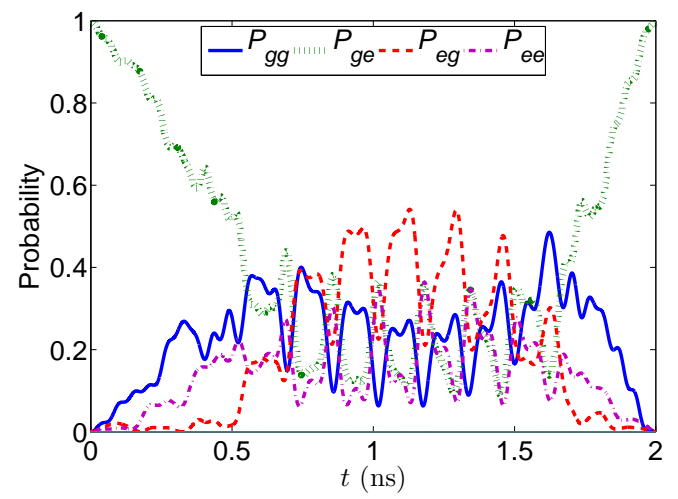

(f)

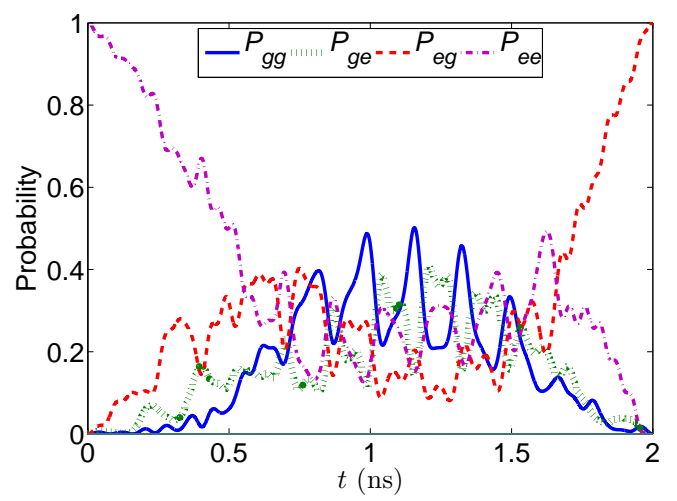

FIG. 4. (Color online) Optimal control sequences and state probability evolutions for a two-qubit CNOT gate with operation time of $T=2.0$ ns. The optimal control pulse sequences of the reduced magnetic fluxes (a) $f_{c}^{(1)}(t)$ and (b) $f_{c}^{(2)}(t)$ are obtained via the Hamiltonian, Eq. (26). The time evolutions of the two-qubit computational-state probabilities calculated via the Hamiltonian, Eq. (2), with the lowest five energy states per qubit using the optimal control sequences shown in (a) and (b) are plotted in (c)-(f) with each two-qubit computational state as an initial state. The two-qubit computational basis states are $|g g\rangle,|g e\rangle,|e g\rangle$ and $|e e\rangle$. 
approximation limits the maximum fidelity (or minimum error) these schemes can actually achieve. One of the common goals for gate control is to implement a two-qubit CNOT gate, an important universal gate for quantum computation. In all of the schemes, an entangled gate natural to their couplings is implemented, and thus to realize a CNOT gate, an additional sequence of single-qubit rotations is needed. For example, if an iSWAP gate is conveniently implemented in a particular scheme, the CNOT gate can be realized by the following sequence CNOT $=\left[I \otimes R_{x}(\pi / 2)\right]\left[R_{z}(-\pi / 2) \otimes R_{z}(\pi / 2)\right][$ iSWAP $]\left[R_{x}(\pi / 2) \otimes\right.$ $I][$ iSWAP $]\left[I \otimes R_{z}(\pi / 2)\right]$. Typical two-qubit gate operation times in these schemes are in the order of 10 to $200 \mathrm{~ns}$. However, decomposing a CNOT gate or a general gate operation into several single-qubit and some entangled two-qubit operations in series makes its operation time normally longer than that of completing the operation in one single run of pulses. Moreover, the overall gate error may also become larger as the gate errors of the decomposed gates will add up or accumulate.

Optimal control approach - In contrast, the optimal control method has great advantages of enabling the implementation of a CNOT gate or other general quantum gates in one single run of pulse or in one single pulse sequence by simply setting the target operation to be the CNOT gate or the general quantum gate one wishes to implement. Requiring the reduced time-dependent magnetic flux $\left|f_{c}^{(l)}(t)\right|=\left|\Phi_{e}^{(l)}(t) / \Phi_{0}\right| \lesssim 10^{-3}$, we then set the CNOT gate operation time $T=2 \mathrm{~ns}$ and use the reduced Hamiltonian (26) to find the optimal control sequence. This CNOT gating time is about several folds to two orders of magnitude shorter than that of the directly or/and indirectly microwave controllable coupling schemes 18, 2633]. This is because single-qubit and two-qubit operations can be performed in parallel simultaneously (rather than in series) in one single run of the optimal control pulses. One can see from Fig. 2 that the CNOT gate error drops quickly with the number of iterations. Here, qubit 1 is the control qubit and qubit 2 is the target qubit. The optimal control sequences obtained when the iteration terminates at the point when error $\eta<10^{-10}$ are shown in Figs. 这(a) and [4]

\section{Effect of leakage states}

To investigate the gate error due to the leakage to higher energy-level states outside the computational basis-state space, we apply the control sequences of the single-qubit and two-qubit gates obtained from the optimization of the reduced two-level-approximation Hamiltonian (26) to the multi-level Hamiltonian (22) and calculate the errors $\eta_{P}$ of Eq. (30) in the projected two-qubit computational state subspace. The single-qubit gate errors are found to be $\eta_{P}=6.56 \times 10^{-8}, 6.21 \times 10^{-8}, 3.25 \times 10^{-8}$, and $5.79 \times 10^{-8}$ for the $Z_{1}, X_{1}, Z_{2}$ and $X_{2}$ gates, respectively. These results indicate that the excitations to higher energy-level states increase slightly the gate errors; however, the gate errors in the order of $10^{-8}$ are still much smaller than $10^{-3}$ of those conventional approaches that apply directly a microwave $\pi$ pulse by neglecting the qubit-qubit interaction. We also simulate the $X_{1}$ gate numerically with the multi-level Hamiltonian (2) using the optimal control sequences obtained from the reduced Hamiltonian (26) for initial four different qubit computational basis states $|g g\rangle,|g e\rangle$, $|e g\rangle$ and $|e e\rangle$. The time evolutions of the state probabilities of the $X_{1}$ gate are shown in

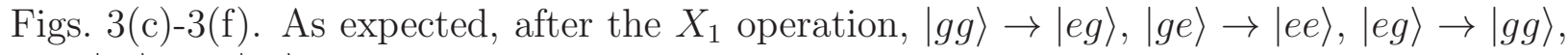
and $|e e\rangle \rightarrow|g e\rangle$. It seems that there is an approximate symmetry under time-reversal

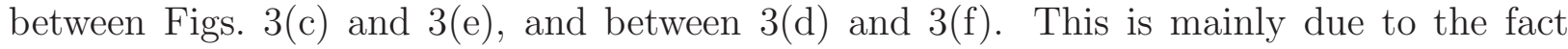
that the control pulse sequences for the $X_{1}$ gate shown in Figs. [3[(a) and [3(b)] are seemingly 
symmetric with respect to the midpoint of the operation time. Thus the time evolutions of the state probabilities, when the first qubit making the transition between $|g\rangle \rightarrow|e\rangle$ and $|e\rangle \rightarrow|g\rangle$ with the second qubit returning back to the same state, appear to have such a time-reversal symmetry. In our case, the pulse sequences are obtained when the gate error is smaller than the error threshold of $\eta<10^{-10}$ for a certain initial guess of the pulse sequence. The truly global optimal pulses should, however, correspond to the minimum value of the cost function obtained by trying all possible initial guesses of the pulse shapes. Thus other initial guesses can generate slightly different $X_{1}$-gate pulse sequences satisfying still the same error threshold requirement but with less symmetry. In fact, the pulse sequence of $f_{c}^{(2)}(t)$ for the $X_{2}$ gate we obtain (not shown here) does not have an apparently symmetric shape with respect to the midpoint of the operation time as compared to $f_{c}^{(1)}(t)$ of the $X_{1}$ gate in Figs. 3. (a), As a result, the time evolutions of the state probability between $|g g\rangle \rightarrow|g e\rangle(|e g\rangle \rightarrow|e e\rangle)$ and $|g e\rangle \rightarrow|g g\rangle(|e e\rangle \rightarrow|e g\rangle)$ for the $X_{2}$ gate (not shown here) do not show prominent time reversal symmetry as their corresponding counterparts for the $X_{1}$ gate. There is, however, indeed an approximate symmetry in time evolutions of the state probabilities in Figs. 3.(c) $3(\mathrm{f})$. This approximate symmetry could be understood in terms of Hamiltonian Eq. (26), or more easily in terms of Hamiltonian Eq. (31) by neglecting the tiny contributions of the last three time-dependent terms of Eq. (26). If one puts aside the fixed static qubit-qubit interaction $\Lambda_{22}$ term, then the two qubits in Hamiltonian Eq. (31) are decoupled and have the symmetry of exchanging $\left|e_{l}\right\rangle$ and $\left|g_{l}\right\rangle$ in the presence of $\sigma_{x}^{(l)}$ term. As a result, the time evolutions of the state probabilities $P_{e g}, P_{e e}, P_{g g}$ and $P_{g e}$ in Figs. [3(c). 3](f), respectively, behave approximately the same. Similarly, the approximately same time evolutions for the state probabilities $P_{g g}, P_{g e}, P_{e g}$ and $P_{e e}$ in Figs. 这(c) ras), respectively, are also observed.

Applying the optimal control sequences of two-qubit CNOT gate shown in Figs. 4) (a) and (b) to multi-level Hamiltonian Eq. (2) with initial four different qubit computational basis states $|g g\rangle,|g e\rangle,|e g\rangle$ and $|e e\rangle$, we plot the time evolutions of the state probabilities of the CNOT gate in Figs. 4l(c) $4(\mathrm{f})$, When qubit 1 is initially in the state $|g\rangle$, the two qubits return to their original states; when qubit 1 is initially in the state $|e\rangle$, an effective NOT operation is performed on qubit 2 (the target qubit) at the end of the CNOT gate operation. This is exactly what we see in Figs. 4](c) 4](f). The error of the CNOT gate when including the higher energy-level states is $\eta_{P} \approx 1.57 \times 10^{-6}$. By Comparing this error to the error of the single-qubit gates of $\eta_{P}<7 \times 10^{-8}$, leakage to the higher-energy level states outside the computational basis state space is more appreciable for the CNOT gate control pulses. This may be due to the fact that the magnitude of the reduced time-dependent magnetic flux $f_{c}^{(2)}(t)$ of Fig. 4 (b) for the CNOT gate is larger than $f_{c}^{(1)}(t)$ and $f_{c}^{(2)}(t)$ for the single-qubit gates [see, e.g., $f_{c}^{(1)}(t)$ and $f_{c}^{(2)}(t)$ of Figs. [3] and [a 3 (b) for the $X_{1}$ gate]. Nevertheless, the CNOT gate error of the two coupled flux qubits is, to our knowledge, still much lower than the currently available schemes when the leakage to higher energy-level states is considered. We have also performed an optimal CNOT gate operation with qubit 1 being the target qubit and qubit 2 being the control qubit. This CNOT gate can also be accomplished at $T=2.0 \mathrm{~ns}$ with error $\eta_{P} \approx 6.60 \times 10^{-7}$. 


\section{Effect of decoherence}

We show below that our optimal control scheme is robust against qubit decoherence. We use the Born-Markov master equation for the reduced density matrix of the flux qubit system in the Lindblad form to model the effect of decoherence on the qubit dynamics [59 61]:

$$
\begin{aligned}
\dot{\rho}(t) & =-\imath[H, \rho(t)]+\sum_{i=1}^{2}\left(\Gamma_{1, i} \mathcal{D}\left[\sigma_{i}^{-}\right] \rho(t)+\Gamma_{\varphi, i} \mathcal{D}\left[\sigma_{i}^{z}\right] \rho(t)\right), \\
& \equiv \mathcal{L} \rho(t),
\end{aligned}
$$

where the Hamiltonian $H$ is defined in Eq. (26), the superoperator

$$
\mathcal{D}[c] \rho=c \rho c^{\dagger}-\frac{1}{2} c^{\dagger} c \rho-\frac{1}{2} \rho c^{\dagger} c
$$

and $\Gamma_{1, i}$ and $\Gamma_{\varphi, i}$ are the relaxation rate and dephasing rate of qubit $i$, respectively. Generally speaking, if one starts from a microscopic model in the weak system-environment coupling limit, the argument in the superoperator $\mathcal{D}$ in the Born-Markov approximation should rather be operators generating transitions between the energy eigenstates of the coupled-qubit Hamiltonian (26) [61]. However, for small inter-qubit coupling considered here, these operators in the rotating-wave approximation can be approximately represented by individual qubit transition operators, $\sigma_{i}^{-}$and $\sigma_{i}^{+}$. In addition, at finite temperatures one should have a dissipative term describing the influx of energy from the thermal environment into the system. But since typical flux-qubit experiments are conducted at a low temperature around $50 \mathrm{mK}$ [62] which is much smaller than the transition frequencies of the qubits considered here (i.e., $\hbar \omega_{l} / k_{B} T \gg 1$ ). As a result, the thermal mean occupation number of the environment modes at the energy of about $\hbar \omega_{l}$ approaches zero, and the environment may be regarded as an effective zero-temperature bath. These justify the use of the master equation (33) to phenomenologically model the decoherence dynamics of the coupled flux-qubit system. The master equation is symbolically expressed in a concise form with a Liouville's superoperator $\mathcal{L}$ in Eq. (34). The equation of motion of the propagator can be found, by substituting $\rho(t)=\mathcal{G}(t) \rho(0)$ into Eq. (34), to be

$$
\dot{\mathcal{G}}(t)=\mathcal{L} \mathcal{G}(t) \text {. }
$$

With the relaxation time $T_{1}=13 \mu$ s obtained from the Rabi oscillation and the decoherence time $T_{2}=2.5 \mu \mathrm{s}$ from Ramsey interference measurements in an recent experiment [62] that probed the noise spectrum of a superconducting flux qubit, one is able to deduce the realistic values of $\Gamma_{1, i}$ and $\Gamma_{\varphi, i}$ using the relations of $\Gamma_{2, i}=\Gamma_{1, i} / 2+\Gamma_{\varphi, i}, \Gamma_{1, i}=1 / T_{1, i}$ and $\Gamma_{2, i}=1 / T_{2, i}$. Define the gate error $\eta_{D}$ by replacing $U(T)$ with $\mathcal{G}(T)$ in Eq. (28) for the case considering qubit decoherence. One can perform the optimal control calculation by minimizing the cost function Eq. (29) with $\eta \rightarrow \eta_{D}$ using the equation of motion (36). The single-qubit gate errors considering the effect of decoherence are found to be in the order of $10^{-6}$, and the errors are in the order of $10^{-5}$ for two-qubit CNOT gates. These gate errors are still below the error threshold $10^{-4}\left(10^{-3}\right.$ in [48]; $10^{-2}$ if surface code error correction is used [49 51]) required for fault-tolerant quantum computation. We have also tested numerically that using a finite-temperature master equation with arguments in the superoperator $\mathcal{D}$ being the operators generating transitions between the energy eigenstates but with the decay rates and the dephasing rates assuming to be the same as individual single qubits leads to the similar error values with correction only to the digits in $10^{-8}$ or $10^{-7}$. 
TABLE I. Summary of the quantum gate errors

\begin{tabular}{|c|c|c|c|c|}
\hline gate & gate time & $\eta$ (unitary) & $\eta_{P}$ (leakage) & $\eta_{D}$ (decoherence) \\
\hline \hline$Z_{1}$ & $0.8 \mathrm{~ns}$ & $<10^{-10}$ & $6.56 \times 10^{-8}$ & $2.46 \times 10^{-6}$ \\
\hline$X_{1}$ & $0.8 \mathrm{~ns}$ & $<10^{-10}$ & $6.21 \times 10^{-8}$ & $2.34 \times 10^{-6}$ \\
\hline$Z_{2}$ & $0.9 \mathrm{~ns}$ & $<10^{-10}$ & $3.25 \times 10^{-8}$ & $3.84 \times 10^{-6}$ \\
\hline$X_{2}$ & $0.9 \mathrm{~ns}$ & $<10^{-10}$ & $5.79 \times 10^{-8}$ & $3.76 \times 10^{-6}$ \\
\hline $\mathrm{CNOT}_{1}$ & $2.0 \mathrm{~ns}$ & $<10^{-10}$ & $6.6 \times 10^{-7}$ & $1.42 \times 10^{-5}$ \\
\hline $\mathrm{CNOT}_{2}$ & $2.0 \mathrm{~ns}$ & $<10^{-10}$ & $1.57 \times 10^{-6}$ & $1.42 \times 10^{-5}$ \\
\hline
\end{tabular}

\section{DISCUSSION AND CONCLUSION}

We have applied the quantum optimal control method to implement fast and high-fidelity single-qubit and two-qubit gates for two inductively coupled superconducting flux qubits with fixed static off-diagonal qubit-qubit coupling and fixed qubit transition frequencies. Table I summarizes the gate errors calculated with realistic experimental parameters for ideal unitary case, and for the cases considering the effect of leakage state and the effect of qubit decoherence. Our optimal control scheme has the following great advantages. (1) Our scheme that shares the same advantage of other directly coupling scheme requires no additional coupler subcircuit and control lines and thus is simple in experimental design. The control lines needed are only for the manipulation of individual qubits (e.g., the timedependent magnetic flux or field through each qubit). (2) Quantum gates constructed via our scheme are all with very high fidelity (very low error) as our optimal control scheme takes into account the fixed qubit detuning and fixed two-qubit interaction as well as all other timedependent magnetic-field-induced single-qubit interactions and two-qubit couplings when performing single-qubit and two-qubit gates. (3) Our scheme can cope with noise and decoherence very well as the qubits are biased at the optimal coherence point to reduce the influence of low-frequency flux noise, and the gate operation time $(\sim 2 \mathrm{~ns})$ is much shorter than the corresponding qubit decoherence time (a few $\mu$ s or larger) [62] so that the decoherence effect on such a fast gate is significantly diminished. (4) A CNOT gate or other general quantum gates can be implemented in a single run of pulse sequence rather than being decomposed into several single-qubit and some entangled two-qubit operations in series by composite pulse sequences.

A natural question then arises: how to generate these optimal control pulses experimentally? The pulse sequences shown in Fig. 3. (a) and (b), and in Fig. 4(a) and (b) look experimentally challenging, but not impossible. Commercial devices (e.g., Tektronix AWG70001A) for generating arbitrary wave forms with 10 bits of vertical resolution at a sample rate of 50 $\mathrm{GSa} / \mathrm{s}$, a bit rate of $12.5 \mathrm{~Gb} / \mathrm{s}$ and a rise/fall time smaller than 27 ps are now available. Such a device should enable generation of complex signals in a time scale of sub-nanoseconds to nanoseconds. This high-end arbitrary wave form generator combining with ultrafast Josephson electronics should be or about to be able to fulfill the necessary specifications for implementing the optimal control pulse sequences we obtain for the quantum gate operations presented here.

Another challenge for the experimental implementations and applications of the quantum optimal control theory is one's imprecise knowledge of the quantum system's parameters. Typically, the quantum gates constructed by the quantum optimal control theory are com- 
puted to very high precision assuming the parameters in the model Hamiltonian are exactly known. However, for real systems controlled in the experiments, the parameters and also the Hamiltonians usually are not known exactly. This poses a challenge to implement such highfidelity gates successfully in the laboratory. Recently, a hybrid open/closed-loop optimal control method called adaptation by hybrid optimal control (Ad-HOC) has been proposed 63. to overcome not only the problem of inaccurate knowledge of the system parameters but also shortcomings of the assumed physical model and errors on the controls themselves. The basic idea is to use the open-loop quantum optimal control theory to find the optimal control pulses with the best available model and parameters of the system. Then the pulses are sent to the experiment and their performance are efficiently measured by using, for example, the method of randomized benchmarking [64 66]. The closed-loop pulse calibration of Ad-HOC, similar to adaptive model-free feedback control (also referred to as closed-loop laboratory control or learning control) [68, 69], uses the physical system itself as a feedback to calibrate control pulses and optimize their performance. By using, e.g., the robust and efficient Nelder-Mead algorithm [63, 66, 67], the control pulses are updated experimentally and the procedure is iterated until a target performance is reached or convergence stops. After the calibration is finished, the optimized pulses can then be used. Even though the precise experimental parameters are never identified, Ad-HOC can reduce the initial gate errors of the numerically obtained optimal control pulses implemented in an experiment by at least an order of magnitude [63]. Thus the quantum optimal control theory is practical and applicable experimentally through, e.g., the Ad-HOC protocol [63]. A recent experiment demonstrating single-qubit operations (with fidelity of 0.99) and two-qubit entanglement for the electron spins of two proximal nitrogen-vacancy centers in diamond using optimal control has been reported [70]. It is thus believed that high-fidelity quantum gates with optimal control pulses obtained via our scheme will be realized experimentally in the near future.

\section{ACKNOWLEDGMENTS}

We are very grateful to Prof. Yu-xi Liu for sending us his note for the derivation of the coupled flux-qubit Hamiltonian of Ref. [28]. We acknowledge support from the National Science Council in Taiwan under Grant No. 100-2112-M-002-003-MY3, from the National Taiwan University under Grants No. 103R891400, No. $103 R 891402$ and 102R3253, and from the focus group program of the National Center for Theoretical Sciences, Taiwan.

[1] J. Q. You and F. Nori, Phys. Today 58, 11, 42 (2005); J. Q. You and F. Nori, Nature 474, 589 (2011), and references therein.

[2] J. Clarke and F. K. Wilhelm, Nature 453, 1031 (2008), and references therein.

[3] Y. Nakamura, Yu. A. Pashkin, and J. S. Tsai, Nature 398, 786 (1999).

[4] D. Vion, A. Aassime, A. Cottet, P. Joyez, H. Pothier, C. Urbina, D. Esteve, and M. H. Devoret, Science 296, 886 (2002).

[5] I. Chiorescu, Y. Nakamura, C. J. P. M. Harmans, and J. E. Mooij, Science 299, 1869 (2003).

[6] P. Bertet, I. Chiorescu, G. Burkard, K. Semba, C. J. P. M. Harmans, D. P. DiVincenzo, and J. E. Mooij, Phys. Rev. Lett. 95, 257002 (2005). 
[7] A. Wallraff, D. I. Schuster, A. Blais, L. Frunzio, J. Majer, M. H. Devoret, S. M. Girvin, and R. J. Schoelkopf, Phys. Rev. Lett. 95, 060501 (2005).

[8] J. M. Martinis, K. B. Cooper, R. McDermott, M. Steffen, M. Ansmann, K. D. Osborn, K. Cicak, S. Oh, D. P. Pappas, R. W. Simmonds, and C. C. Yu, Phys. Rev. Lett. 95, 210503 (2005).

[9] F. Yoshihara, K. Harrabi, A. O. Niskanen, Y. Nakamura, and J. S. Tsai, Phys. Rev. Lett. 97, 167001 (2006).

[10] K. Kakuyanagi, T. Meno, S. Saito, H. Nakano, K. Semba, H. Takayanagi, F. Deppe, and A. Shnirman, Phys. Rev. Lett. 98, 047004 (2007).

[11] A. D. Córcoles, J. M. Chow, J. M. Gambetta, C. Rigetti, J. R. Rozen, G. A. Keefe, M. B. Rothwell, M. B. Ketchen, and M. Steffen, Appl. Phys. Lett. 99, 181906 (2011).

[12] C. Rigetti, J. M. Gambetta, S. Poletto, B. L. T. Plourde, J. M. Chow, A. D. Córcoles, J. A. Smolin, S. T. Merkel, J. R. Rozen, G. A. Keefe, M. B. Rothwell, M. B. Ketchen, and M. Steffen, Phys. Rev. B 86, 100506 (2012).

[13] J. M. Chow, L. DiCarlo, J. M. Gambetta, F. Motzoi, L. Frunzio, S. M. Girvin, and R. J. Schoelkopf, Phys. Rev. A 82, 040305(R) (2010).

[14] H. Paik, D. I. Schuster, L. S. Bishop, G. Kirchmair, G. Catelani, A. P. Sears, B. R. Johnson, M. J. Reagor, L. Frunzio, L. I. Glazman, S. M. Girvin, M. H. Devoret, and R. J. Schoelkopf, Phys. Rev. Lett. 107, 240501 (2011).

[15] T. Yamamoto, Yu. A. Pashkin, O. Astafiev, Y. Nakamura, and J. S. Tsai, Nature 425, 941 (2003).

[16] A. Sillanpää, J. I. Park, and R. W. Simmonds, Nature 449, 438 (2007).

[17] J. Majer, J. M. Chow, J. M. Gambetta, J. Koch, B. R. Johnson, J. A. Schreier, L. Frunzio, D. I. Schuster, A. A. Houck, A. Wallraff, A. Blais, M. H. Devoret, S. M. Girvin, and R. J. Schoelkopf, Nature 449, 443 (2007).

[18] A. O. Niskanen, K. Harrabi, F. Yoshihara, Y. Nakamura, S. Lloyd, and J. S. Tsai, Science 316, 723 (2007).

[19] J. H. Plantenberg, P. C. de Groot, C. J. P. M. Harmans, and J. E. Mooij, Nature 447, 836 (2007).

[20] L. DiCarlo, J. M. Chow, J. M. Gambetta, L. S. Bishop, B. R. Johnson, D. I. Schuster, J. Majer, A. Blais, L. Frunzio, S. M. Girvin, and R. J. Schoelkopf, Nature 460, 240 (2009).

[21] J. M. Chow, A. D. Córcoles, J. M. Gambetta, C. Rigetti, B. R. Johnson, J. A. Smolin, J. R. Rozen, G. A. Keefe, M. B. Rothwell, M. B. Ketchen, and M. Steffen, Phys. Rev. Lett. 107, 080502 (2011).

[22] A. Dewes, F. R. Ong, V. Schmitt, R. Lauro, N. Boulant, P. Bertet, D. Vion, and D. Esteve, Phys. Rev. Lett. 108, 057002 (2012).

[23] J. M. Chow, J. M. Gambetta, A. D. Córcoles, S. T. Merkel, J. A. Smolin, C. Rigetti, S. Poletto, G. A. Keefe, M. B. Rothwell, J. R. Rozen, M. B. Ketchen, and M. Steffen, Phys. Rev. Lett. 109, 060501 (2012).

[24] L. DiCarlo, M. D. Reed, L. Sun, B. R. Johnson, J. M. Chow, J. M. Gambetta, L. Frunzio, S. M. Girvin, M. H. Devoret, and R. J. Schoelkopf, Nature 467, 574 (2010); M. Neeley, R. C. Bialczak, M. Lenander, E. Lucero, M. Mariantoni, A. D. O’Connell, D. Sank, H. Wang, M. Weides, J. Wenner, Y. Yin, T. Yamamoto, A. N. Cleland, and J. M. Martinis, Nature 467, $570(2010)$.

[25] X.-Y. Lü, S. Ashhab, W. Cui, R. Wu, and F. Nori, New J. Phys. 14, 073041 (2012).

[26] C. Rigetti, A. Blais, and M. Devoret, Phys. Rev. Lett. 94, 240502 (2005). 
[27] P. Bertet, C. J. P. M. Harmans, and J. E. Mooij, Phys. Rev. B 73, 064512 (2006).

[28] Y.-X. Liu, L. F. Wei, J. S. Tsai, and F. Nori, Phys. Rev. Lett. 96, 067003 (2006).

[29] S. Ashhab, S. Matsuo, N. Hatakenaka, and F. Nori, Phys. Rev. B 74, 184504 (2006); S. Ashhab and F. Nori, Phys. Rev. B 76, 132513 (2007).

[30] C. Rigetti and M. Devoret, Phys. Rev. B 81, 134507 (2010).

[31] A. O. Niskanen, Y. Nakamura, and J. S. Tsai, Phys. Rev. B 73, 094506 (2006).

[32] K. Harrabi, F. Yoshihara, A. O. Niskanen, Y. Nakamura, and J. S. Tsai, Phys. Rev. B 79, 020507(R) (2009).

[33] M. Grajcar, Y.-X. Liu, F. Nori, and A. M. Zagoskin, Phys. Rev. B 74, 172505 (2006).

[34] A. P. Peirce, M. A. Dahleh, and H. Rabitz, Phys. Rev. A 37, 4950 (1988).

[35] D. J. Tannor, V. A. Kazakov and V. Orlov, in Time-Dependent Quantum Molecular Dynamics, edited by J. Broeckhove and L. Lathouwers, NATO Advanced Studies Institute, Series B: Physics (Plenum Press, New York, 1992), Vol. 299, pp. 347-360.

[36] J. P. Palao and R. Kosloff, Phys. Rev. Lett. 89, 188301 (2002); J. P. Palao and R. Kosloff, Phys. Rev. A 68, 062308 (2003).

[37] N. Khaneja, T. Reiss, C. Kehlet, T. Schulte-Herbrüggen, and S. J. Glaser, J. Magn. Reson. 172, 296 (2005); D.-B. Tsai, P.-W. Chen, and H.-S. Goan, Phys. Rev. A 79, 060306(R) (2009).

[38] A. Spörl, T. Schulte-Herbrüggen, S. J. Glaser, V. Bergholm, M. J. Storcz, J. Ferber, and F. K. Wilhelm, Phys. Rev. A 75, 012302 (2007).

[39] R. Eitan, M. Mundt, and D. J. Tannor, Phys. Rev. A 83, 053426 (2011).

[40] S. Montangero, T. Calarco, and R. Fazio, Phys. Rev. Lett. 99, 170501 (2007).

[41] I. I. Maximov, Z. Tošner, and N. C. Nielsen, J. Chem. Phys. 128, 184505 (2008).

[42] M. Wenin and W. Pötz, Phys. Rev. A 78, 012358 (2008); M. Wenin and W. Pötz, Phys. Rev. B 78, 165118 (2008); M. Wenin, R. Roloff, and W. Pötz, J. Appl. Phys. 105, 084504 (2009).

[43] R. Roloff and W. Pötz, Phys. Rev. B 79, 224516 (2009); M. Wenin and W. Pötz, Phys. Rev. A 74, 022319 (2006).

[44] H. Jirari, Europhys. Lett. 87, 40003 (2009).

[45] P. Rebentrost, I. Serban, T. Schulte-Herbrüggen, and F. K. Wilhelm, Phys. Rev. Lett. 102, 090401 (2009).

[46] B. Hwang and H.-S. Goan, Phys. Rev. A 85, 032321 (2012).

[47] J.-S. Tai, K.-T. Lin and H.-S. Goan, Phys. Rev. A 89, 062310 (2014).

[48] P. Aliferis and J. Preskill, Phys. Rev. A 79, 012332 (2009).

[49] D. S. Wang, A. G. Fowler, and L. C. L. Hollenberg, Phys. Rev. A 83, 020302(R) (2011).

[50] A. G. Fowler, A. C. Whiteside, and L. C. L. Hollenberg, Phys. Rev. Lett. 108, 180501 (2012),

[51] A. G. Fowler, M. Mariantoni, J. M. Martinis, A. N. Cleland, Phys. Rev. A 86, 032324 (2012)

[52] J. B. Majer, F. G. Paauw, A. C. J. ter Haar, C. J. P. M. Harmans, and J. E. Mooij, Phys. Rev. Lett. 94, 090501 (2005), and references therein.

[53] T. P. Orlando, J. E. Mooij, L. Tian, C. H. van der Wal, L. S. Levitov, S. Lloyd, and J. J. Mazo, Phys. Rev. B 60, 15398 (1999).

[54] Y.-X. Liu, J. Q. You, L. F. Wei, C. P. Sun, and F. Nori, Phys. Rev. Lett. 95, 087001 (2005).

[55] F. Deppe, M. Mariantoni, E. P. Menzel, A. Marx, S. Saito, K. Kakuyanagi, H. Tanaka, T. Meno, K. Semba, H. Takayanagi, E. Solano, and R. Gross, Nat. Phys. 4, 686 (2008).

[56] V. F. Krotov, Global Methods in Optimal Control Theory (Marcel Dekker, New York, 1996).

[57] A. Izmalkov, M. Grajcar, E. Il'ichev, Th. Wagner, H.-G. Meyer, A. Yu. Smirnov, M. H. S. Amin, A. Maassen van den Brink, and A. M. Zagoskin, Phys. Rev. Lett. 93, 037003 (2004).

[58] J. Q. You, Y. Nakamura, and F. Nori, Phys. Rev. B 71, 024532 (2005). 
[59] H. J. Carmichael, Statistical Methods in Quantum Optics 1 (Springer, Berline, 1999).

[60] C. W. Gardiner and P. Zoller, Quantum Noise, 2nd edition. (Springer-Verlag, Berlin, 2000).

[61] H.P. Breuer and F. Petruccione, The Theory of Open Quantum Systems (Oxford University Press, Oxford, 2002).

[62] J. Bylander, S. Gustavsson, F. Yan, F. Yoshihara, K. Harrabi, G. Fitch, D. G. Cory, Y. Nakamura, J. S. Tsai, and W. D. Oliver, Nat. Phys. 7, 565-570 (2011).

[63] D. J. Egger, and F. K. Wilhelm, arXiv:1402.7193.

[64] E. Magesan, J. M. Gambetta, B. R. Johnson, C. A. Ryan, J. M. Chow, S. T. Merkel, M. P. da Silva, G. A. Keefe, M. B. Rothwell, T. A. Ohki, M. B. Ketchen, and M. Steffen, Phys. Rev. Lett. 109, 080505 (2012); E. Magesan, J. M. Gambetta, and J. Emerson, Phys. Rev. Lett. 106, 180504 (2011).

[65] J. M. Chow, J. M. Gambetta, L. Tornberg, J. Koch, L. S. Bishop, A. A. Houck, B. R. Johnson, L. Frunzio, S. M. Girvin, and R. J. Schoelkopf, Phys. Rev. Lett. 102, 090502 (2009);

[66] J. Kelly, R. Barends et al., arXiv:1403.0035.

[67] J. A. Nelder and R. Mead, Comput. J. 7, 308 (1967).

[68] R. S. Judson and H. Rabitz, Phys. Rev. Lett. 68, 1500 (1992).

[69] C. Brif, R. Chakrabarti1 and H. Rabitz, New J. Phys. 12, 075008 (2010).

[70] F. Dolde, V. Bergholm, Y. Wang, I. Jakobi, S. Pezzagna, J. Meijer, P. Neumann, T. SchulteHerbrüggen J. Biamonte, and J. Wrachtrup, Nat. Commun. 5, 3371 (2014). 